MATHEMATICS OF COMPUTATION

Volume 66, Number 219, July 1997, Pages 1073-1087

S $0025-5718(97) 00850-8$

\title{
ESTIMATING THE NUMBER OF ASYMPTOTIC DEGREES OF FREEDOM FOR NONLINEAR DISSIPATIVE SYSTEMS
}

\author{
BERNARDO COCKBURN, DON A. JONES, AND EDRISS S. TITI
}

\begin{abstract}
We show that the long-time behavior of the projection of the exact solutions to the Navier-Stokes equations and other dissipative evolution equations on the finite-dimensional space of interpolant polynomials determines the long-time behavior of the solution itself provided that the spatial mesh is fine enough. We also provide an explicit estimate on the size of the mesh. Moreover, we show that if the evolution equation has an inertial manifold, then the dynamics of the evolution equation is equivalent to the dynamics of the projection of the solutions on the finite-dimensional space spanned by the approximating polynomials. Our results suggest that certain numerical schemes may capture the essential dynamics of the underlying evolution equation.
\end{abstract}

\section{INTRODUCTION}

Physical arguments indicate that the asymptotic behavior of the solutions of certain dissipative evolution equations can be described by only a finite number of degrees of freedom. Such equations include, but are not limited to, systems of reaction-diffusion equations as well as systems that arise in fluid mechanics. See for example [24], [26]. This assertion was first rigorously proven by [10], in the case of the two-dimensional Navier-Stokes equations (NSE). Specifically, they show that if the difference between the first $N$ Fourier modes of any two solutions of the Navier-Stokes equations tends to zero, asymptotically in time as time goes to infinity, for $N$ sufficiently large, then the difference between the two solutions tends to zero, in an appropriate norm, as time goes to infinity. An explicit estimate on $N$, in terms of the Reynolds number, was first given by Foias, Manley, Temam and Treve [8] and later improved by Jones and Titi [22].

After this rigorous pioneering work of Foias and Prodi [10] several authors proved similar results for other degrees of freedom than the components of the Fourier modes. Foias and Temam [14] used the values of the solutions at nodes in the spatial domain as degrees of freedom. The work [15] and later [21] used local averages of the solutions on finite volumes as the degrees of freedom. Explicit estimates for these kinds of degrees of freedom were presented in [22]. In this paper, we further extend the above results and show that for a large class of dissipative evolution equations, including the Navier-Stokes equations, there is a larger collection of determining sets of degrees of freedom (see Definition 1.1 below), than the ones mentioned above. The existence of such a collection was asserted in [13]. Part of the results of this paper have been announced in [3].

Received by the editor July 27, 1995 and, in revised form, June 5, 1996.

1991 Mathematics Subject Classification. Primary 35B40, 35Q30.

(C)1997 American Mathematical Society 
Definition 1.1. Let $\left\{\ell_{i}\right\}_{i=1}^{N}$ be a set of bounded linear functionals on the space of solutions for a given evolution dissipative PDE. Suppose that whenever $\lim _{t \rightarrow \infty} \ell_{i}\left(u_{1}(t)-u_{2}(t)\right)=0$, for $1 \leq i \leq N$, we conclude that $\lim _{t \rightarrow \infty}\left\|u_{1}(t)-u_{2}(t)\right\|$ $=0$, where $u_{1}$ and $u_{2}$ are any two solutions. Then we say that $\left\{\ell_{i}\right\}_{i=1}^{N}$ is a set of determining functionals.

These determining functionals can be thought of as determining degrees of freedom for the long-time dynamics of the PDE.

In the case the underlying dissipative evolution equation possesses an inertial manifold we can extend and sharpen these results. In particular, following the work of Foias and Titi [15], we show that if a sufficiently large number $N$ of degrees of freedom is taken, then one can parameterize the inertial manifold in terms of these functionals $\left\{l_{i}\right\}_{i=1}^{N}$. We add that, in this case, one can choose the number $N$ to be comparable with the dimension of the inertial manifold. Moreover, in this case we show that if the degrees of freedom of any two solutions on the inertial manifold coincide at one instant, then the solutions agree for all nonnegative time.

The paper is organized as follows. In Section 2, we state, discuss, and prove our results concerning determining degrees of freedom for the two-dimensional NavierStokes equations on a square with periodic boundary conditions. We want to point out that the results of Theorem 2.1 and the techniques used to prove it also hold in the case of two-dimensional Navier-Stokes equations subject to the no-slip Dirichlet boundary condition. However, in this case one obtains larger upper bounds for the number of degrees of freedom. Whether this is a mathematical technicality or is due to the effects of physical boundaries remains to be explored. In Section 3, we display and discuss our results about determining degrees of freedom for general nonlinear dissipative evolution equations that possess an inertial manifold. In particular, we show that the inertial manifold can be parameterized by these determining degrees of freedom. On the other hand as we will see in Section 2, for the case of the NSE, the notion of determining degrees of freedom is not restricted to equations that possess an inertial manifold (the existence of an inertial manifold for the NSE is still an open question). In Section 4 we conclude by discussing conditions on general dissipative evolution equations that guarantee the existence of a set of determining degrees of freedom for these equations.

Our results suggest that the long-time behavior of the solutions may be captured by the dynamics of the projections on these finite-dimensional approximating spaces. Indeed, in the case where an inertial manifold exists, one shows that the dynamics of the underlying equation is equivalent to the dynamics of the projections (Section 3). The real question is: does the dynamics of the approximating discretization approximate that of the underlying equation? We do not attempt to answer this question here. However, our results suggest that the answer of this question might be affirmative. We refer the reader to [17], [5.5], [32], [33], [18], [19] and references therein.

\section{Sets of Determining Degrees of Freedom for the 2D NSE}

2.1. Preliminaries and statement of main result. In this section we consider the problem of finding sets of determining degrees of freedom for the twodimensional NSE for a viscous incompressible fluid on the square $\Omega=(0, L) \times(0, L)$ 
with periodic boundary conditions:

$$
\left\{\begin{array}{c}
\frac{\partial u}{\partial t}-\nu \Delta u+(u \cdot \nabla) u+\nabla p=f \text { in } \mathbb{R}^{2} \times(0, \infty) \\
\nabla \cdot u=0 \text { in } \mathbb{R}^{2} \times(0, \infty) \\
u\left(x_{1}, x_{2}, t\right)=u\left(x_{1}, x_{2}+L, t\right) \\
u\left(x_{1}, x_{2}, t\right)=u\left(x_{1}+L, x_{2}, t\right)
\end{array}\right.
$$

where the volume force $f=f(x, t)$ and the kinematic viscosity $\nu>0$ are given. The velocity vector $u=u(x, t)$ and the pressure $p=p(x, t)$ are the unknowns.

We can rewrite the above equations in terms of the velocity $u$ only as follows. Using the standard notation (see, e.g., [4], [27], [30]), we set

$$
\begin{aligned}
\mathcal{V}= & \left\{u: \mathbb{R}^{2} \rightarrow \mathbb{R}^{2},\right. \text { vector-valued trigonometric polynomials } \\
& \text { with period } \left.L, \nabla \cdot u=0, \text { and } \int_{\Omega} u d x=0\right\}, \\
H= & \text { the closure of } \mathcal{V} \text { in }\left(L^{2}(\Omega)\right)^{2}, \\
V= & \text { the closure of } \mathcal{V} \text { in }\left(H^{1}(\Omega)\right)^{2},
\end{aligned}
$$

where $H^{l}(\Omega) \quad(l=1,2, \ldots)$ denote the usual $L^{2}$-Sobolev spaces. The space $H$ is a Hilbert space with inner product and norm

$$
(u, v)=\int_{\Omega} u(x) \cdot v(x) d x, \quad|u|=\left(\int_{\Omega}|u(x)|^{2} d x\right)^{1 / 2},
$$

respectively, where $u(x) \cdot v(x)$ is the usual Euclidean scalar product. Thanks to the Poincaré inequality, $V$ is also a Hilbert space with the $\left(L^{2}(\Omega)\right)^{2}$ inner product and norm

$$
((u, v))=\sum_{i, j=1}^{2} \int_{\Omega} \frac{\partial u_{i}}{\partial x_{j}} \frac{\partial v_{i}}{\partial x_{j}} d x, \quad\|v\|^{2}=\sum_{i, j=1}^{2} \int_{\Omega}\left|\frac{\partial v_{i}}{\partial x_{j}}\right|^{2} d x,
$$

respectively.

Let $P$ denote the orthogonal projection in $L^{2}(\Omega) \times L^{2}(\Omega)$ onto $H$. We denote by $A$ the Stokes operator

$$
A u=-P \Delta u,
$$

(notice that in the periodic case $A u=-\Delta u$ ) and the bilinear operator

$$
B(u, v)=P((u \cdot \nabla) v)
$$

for all $u, v \in V$ and in this case $B(u, v) \in V^{\prime}$, where $V^{\prime}$ denotes the dual space of $V$. We recall that the operator $A$ is a self-adjoint positive definite operator with compact inverse. Thus there exists a complete orthonormal set $w_{j}$ of eigenfunctions of $A$ such that $A w_{j}=\lambda_{j} w_{j}$ and $0<\lambda_{1} \leq \lambda_{2} \leq \ldots$. Moreover, we have that $\lambda_{1}=\left(\frac{2 \pi}{L}\right)^{2}$, and $\lambda_{j}=O(j)$ for $j \rightarrow \infty$.

We may rewrite the 2D NSE as the evolution equation

$$
\frac{d u}{d t}+\nu A u+B(u, u)=f
$$

on the Hilbert space $H$. We assume that $f=P f$ and that $f$ belongs $L^{\infty}((0, \infty) ; H)$, that is,

$$
\sup _{t \geq 0}|f(t)|<\infty
$$


This hypothesis allows us to introduce the so-called generalized Grashof number $G r$; see [8], defined as follows:

$$
G r=\frac{F}{\lambda_{1} \nu^{2}}=\frac{L^{2} F}{4 \pi^{2} \nu^{2}}
$$

where

$$
F=\limsup _{t \rightarrow \infty}|f(t)| .
$$

Note that if $f$ is time independent, then $G r$ is the Grashof number $G=\frac{L^{2}|f|}{4 \pi^{2} \nu^{2}}$.

Next, we focus our attention on the type of degrees of freedom we want to consider. Since our goal is to compare the asymptotic behavior, as time $t \rightarrow \infty$, of two solutions of the NSE equations (2.1) in terms of the long time behavior of their degrees of freedom, it is reasonable to consider degrees of freedom with which it is possible to reconstruct a good approximation of the original functions. More precisely, given an arbitrary set of bounded linear functionals (degrees of freedom) $\left\{\ell_{i}\right\}_{i=1}^{N}$, defined on $\mathcal{D}(A)$, we consider 'reconstruction' operators $R^{h}$ of the form

$$
R^{h}(u)=\sum_{i=1}^{N} \ell_{i}(u) \phi_{i},
$$

where $\phi_{i} \in\left(L^{2}(\Omega)\right)^{2}$, such that

$$
\left|u-R^{h}(u)\right| \leq c_{1} h^{2}|A u| .
$$

Here $h$ and $N$ are not independent parameters. We use them interchangeably with the understanding that as $h \rightarrow 0, N \rightarrow \infty$ and conversely. Note that although we do not require the functions $\phi_{i}$ to belong to the space $H$, we do require the operator $R^{h}$ to be a good approximation of the inclusion map from $\mathcal{D}(A)$ into $\left(L^{2}(\Omega)\right)^{2}$. In fact, the only property of the operator $R^{h}$ that is used in our analysis is the approximation inequality (2.4). This means that we should not talk about determining degrees of freedom but of determining operators (projections) $R^{h}$; we kept the term degrees of freedom for historical reasons. These abstract assumptions on the operator $R^{h},(2.3),(2.4)$ have been inspired by our previous work on the determining modes, nodes, and volume elements, [22].

Typically, the operator $R^{h}$ is an interpolation operator or a projection operator. Classical examples are constructed as follows. Let $\mathcal{T}^{h}$ be a triangulation, made of triangles, of the domain $\Omega$ and let $\left\{x_{i}\right\}_{i=1}^{N}$ be the set of all the vertices of the triangles $T \in \mathcal{T}^{h}$. Let $V^{h}$ be the set of continuous functions with values in $\mathbb{R}^{2}$ whose restrictions to each triangle $T \in \mathcal{T}^{h}$ is affine in each component. Then, take $\phi_{i}$ to be the element of $V^{h}$ such that $\phi_{i}\left(x_{j}\right)=\delta_{i j}$. We can define $R^{h}$ as an interpolation operator if we take $\ell_{i}(u)=u\left(x_{i}\right)$ (note that the degrees of freedom $\ell_{i}$ are well defined for functions $u \in \mathcal{D}(A)$ since, by elliptic regularity and Sobolev's inequality respectively, $\left.\mathcal{D}(A) \subset\left(H^{2}(\Omega)\right)^{2} \subset\left(C^{0}(\bar{\Omega})\right)^{2}\right)$. As another example we can also define $R^{h}$ as the $L^{2}$-projection of $u$ into $V^{h}$, that is, $R^{h}(u)$ is the only element in $V^{h}$ such that

$$
\left(R^{h}(u), v_{h}\right)=\left(u, v_{h}\right), \quad \forall v_{h} \in V^{h} .
$$

Both of the operators $R^{h}$ defined above satisfy (2.4) with $h$ equal to the maximum of the diameters of the triangles $T \in \mathcal{T}^{h}$. In general, if the space $\operatorname{span}\left\{\phi_{i}, 1 \leq i \leq N\right\}$ includes $V^{h}$ and if $R^{h}\left(v_{h}\right)=v_{h}$ for every $v_{h} \in V^{h}$, then the inequality (2.4) holds. See, for example, [2], [16] and [34]. 
We are now ready to state the main result of this section.

Theorem 2.1. Let $u$ and $v$ be the solutions of the following initial value problems for the $2 D N S E$,

$$
\begin{aligned}
& \frac{d u}{d t}+\nu A u+B(u, u)=f(t), \quad u(0)=u_{0}, \\
& \frac{d v}{d t}+\nu A v+B(v, v)=g(t), \quad v(0)=v_{0}
\end{aligned}
$$

respectively, where $f$ and $g$ are such that

$$
|f(t)-g(t)| \rightarrow 0 \text { as } t \rightarrow \infty .
$$

Suppose that (2.3), (2.4) hold and that

$$
\lim _{t \rightarrow \infty} \ell_{i}(u(t)-v(t))=0, \quad 1 \leq i \leq N .
$$

Then the set of degrees of freedom $\left\{\ell_{i}\right\}_{i=1}^{N}$ is determining for the NSE (2.1), that is,

$$
\lim _{t \rightarrow \infty}\|u(t)-v(t)\|=0
$$

provided

$$
h<h_{0}:=\left(\sqrt{2 c_{2}} c_{1} \lambda_{1} G r\right)^{-1 / 2} .
$$

Here $c_{1}$ is the constant in the approximation inequality (2.4), and $c_{2}$ is the constant of Agmon's inequality;

$$
\|u\|_{L^{\infty}(\Omega)} \leq c_{2}|u|^{\frac{1}{2}}|A u|^{\frac{1}{2}}, \quad \forall u \in \mathcal{D}(A) .
$$

(The constant $c_{2}$ may be bounded by $(10+4 \sqrt{2}) / \pi$ in the case of peroidic boundary conditions.)

Corollary 2.2. Suppose that $N=c_{3} L^{2} h^{-2}$. Then the set of degrees of freedom $\left\{\ell_{i}\right\}_{i=1}^{N}$ is determining for the NSE (2.1) provided

$$
N>4 \pi^{2} \sqrt{2 c_{2}} c_{1} c_{3} G r .
$$

We recall that the best known upper bound for the fractal dimension of the attractor given in [6] is of the order $G^{2 / 3}(1+\log (G))^{1 / 3}$. This estimate agrees up to the logarithmic term with the number of degrees of freedom predicted by physical arguments, and a rigorous lower bound for the Hausdorff dimension of the attractor derived by [1] for this case (see also [28]). The estimate in the corollary is consistent with the bound we derived for the upper bound for the number of determining nodes, determining finite volume elements, and determining modes which are all of the order $G r$, [22]. Alternatively, as discussed in [6] one can deduce that the Kraichnan dissipative micro scale of the enstrophy, [24], is of the order $L G^{-1 / 3}$. Whereas Theorem 2.1 indicates that for this kind of explicit degrees of freedom the size of mesh (of this micro scale) should of the order $h \leq C L G r^{-1 / 2}$. 
2.2. Proof of Theorem 2.1. In this section, we prove Theorem 2.1. We start with several auxiliary lemmas.

Lemma 2.3. (Properties of B.) We have

$$
(B(u, w), A w)+(B(w, u), A w)+(B(w, w), A u)=0, \quad \forall u, w \in \mathcal{D}(A) .
$$

Proof. The first inequality follows immediately from the definition of $B$ (for the proof see for example [4] or [30]). The second equality follows from a straightforward computation using the fact that we are considering the NSE in the two-dimensional space with periodic boundary conditions. The property (2.9) is not known to hold for the general no-slip boundary conditions. The last equality, (2.10), follows by taking the Fréchet derivative of the second inequality, (2.9), in the direction of $u$. Again this a property of the two-dimensional NSE with periodic boundary conditions; see [4].

Lemma 2.4. For every $w \in D(A)$ we have

$$
\begin{aligned}
|w| & \leq\left|R^{h} w\right|+c_{1} h^{2}|A w|, \\
\|w\|^{2} & \leq c_{4} h^{-2}\left|R^{h} w\right|^{2}+c_{5} h^{2}|A w|^{2}, \\
\|w\|_{\infty}^{2}=\sup _{x \in \Omega}|w(x)|^{2} & \leq c_{2} c_{4} h^{-2}\left|R^{h} w\right|^{2}+c_{2} c_{5} h^{2}|A w|^{2},
\end{aligned}
$$

where $c_{4}=1 /\left(2 c^{\prime}\right), c_{5}=\left(1+c^{\prime} / 2\right) c_{1}$, and $c^{\prime}$ is an arbitrary positive number.

Proof. The first inequality follows directly from inequality (2.4). The second inequality follows from the interpolation inequality $\|w\|^{2} \leq|w||A w|$ and from a simple use of Young's inequality. The third inequality follows in a similar way from Agmon's inequality (2.7), namely, $\|w\|_{\infty}^{2} \leq c_{2}|w||A w|$.

Lemma 2.5. For $T=\left(\nu \lambda_{1}\right)^{-1}$, we have

$$
\limsup _{t \rightarrow \infty} \frac{1}{T} \int_{t}^{t+T}|A u|^{2} d \tau \leq 2 \frac{F^{2}}{\nu^{2}}
$$

For a proof, see [8]; see also [20].

The following version of Gronwall's Lemma, obtained by [21], is a generalization of an earlier result by Foias et al. [8].

Lemma 2.6. Let $\alpha$ be a locally integrable real valued function on $(0, \infty)$, satisfying for some $0<T<\infty$ the following conditions:

$$
\liminf _{t \rightarrow \infty} \frac{1}{T} \int_{t}^{t+T} \alpha(\tau) d \tau=\gamma>0, \quad \limsup _{t \rightarrow \infty} \frac{1}{T} \int_{t}^{t+T} \alpha^{-}(\tau) d \tau=\Gamma<\infty,
$$

where $\alpha^{-}=\max \{-\alpha, 0\}$. Further, let $\beta$ be a real valued locally integrable function defined on $(0, \infty)$ such that

$$
\lim _{t \rightarrow \infty} \frac{1}{T} \int_{t}^{t+T} \beta^{+}(\tau) d \tau=0
$$


where $\beta^{+}=\max \{\beta, 0\}$. Suppose that $\xi$ is an absolutely continuous non-negative function on $(0, \infty)$ such that

$$
\frac{d}{d t} \xi+\alpha \xi \leq \beta, \text { a.e. on }(0, \infty) \text {. }
$$

Then $\xi(t) \rightarrow 0$ as $t \rightarrow \infty$.

We are now ready to prove Theorem 2.1.

Set $w(t)=u(t)-v(t)$. Subtracting equations (2.6) and (2.5), we find that

$$
\frac{d w}{d t}+\nu A w+B(u, w)+B(w, u)-B(w, w)=0 .
$$

Upon using (2.9) and (2.10) we obtain that

$$
\frac{1}{2} \frac{d}{d t}\|w\|^{2}+\nu|A w|^{2} \leq|(B(w, w), A u)|+|f-g||A w| .
$$

Using (2.8) and the above we obtain

$$
\frac{1}{2} \frac{d}{d t}\|w\|^{2}+\nu|A w|^{2} \leq\|w\|_{\infty}\|w\||A u|+|f-g \| A w| .
$$

Let $\epsilon>0$. Then using Young's inequality and (2.12), we have that

$$
\begin{aligned}
\|w\|_{\infty}\|w\||A u| & \leq \frac{\epsilon}{2}\|w\|_{\infty}^{2}+\frac{1}{2 \epsilon}\|w\|^{2}|A u|^{2} \\
& \leq \frac{\epsilon c_{2}}{2}\left\{c_{4} h^{-2}\left|R^{h} w\right|^{2}+c_{5} h^{2}|A w|^{2}\right\}+\frac{1}{2 \epsilon}\|w\|^{2}|A u|^{2} .
\end{aligned}
$$

With $\epsilon=\nu c /\left(c_{2} c_{5} h^{2}\right)$ we find that

$$
\|w\|_{\infty}\|w\||A u| \leq=\frac{\nu c c_{4}}{2 c_{5} h^{4}}\left|R^{h} w\right|^{2}+\frac{\nu c}{2}|A w|^{2}+\frac{c_{2} c_{5} h^{2}}{2 \nu c}\|w\|^{2}|A u|^{2} .
$$

Similarly, by using Young's inequality, we get

$$
\begin{aligned}
|f-g||A w| & \leq \frac{\epsilon^{\prime}}{2}|f-g|^{2}+\frac{1}{2 \epsilon^{\prime}}|A w|^{2} \\
& =\frac{1}{2 \nu c^{\prime}}|f-g|^{2}+\frac{\nu c^{\prime}}{2}|A w|^{2},
\end{aligned}
$$

for $\epsilon^{\prime}=1 /\left(\nu c^{\prime}\right)$.

Using the two last inequalities, we obtain, after reordering terms,

$$
\begin{aligned}
\frac{d}{d t}\|w\|^{2} \leq & -|A w|^{2} \nu\left(2-c-c^{\prime}\right)+\frac{c_{2} c_{5} h^{2}}{\nu c}|A u|^{2}\|w\|^{2} \\
& +\frac{\nu c c_{4}}{c_{5} h^{4}}\left|R^{h} w\right|^{2}+\frac{1}{\nu c^{\prime}}|f-g|^{2} .
\end{aligned}
$$

Finally, assuming that

$$
2-c-c^{\prime}>0,
$$

and using (2.12) in the following form,

$$
|A w|^{2} \geq \frac{1}{c_{5} h^{2}}\|w\|^{2}-\frac{c_{4}}{c_{5} h^{4}}\left|R^{h} w\right|^{2},
$$

we get

$$
\frac{d}{d t}\|w\|^{2}+\alpha(t)\|w\|^{2} \leq \beta(t)
$$


where

and

$$
\alpha(t)=\frac{2-c^{\prime}-c}{c_{5} h^{2}} \nu-\frac{c_{2} c_{5} h^{2}}{\nu c}|A u|^{2}
$$

$$
\beta(t)=\frac{\nu\left(2-c^{\prime}\right) c_{4}}{c_{5} h^{4}}\left|R^{h} w\right|^{2}+\frac{1}{\nu c^{\prime}}|f(t)-g(t)|^{2} .
$$

By using the hypotheses on the asymptotic behavior of $\left|R^{h}(w)(t)\right|$ and $\mid f(t)-$ $g(t) \mid$ as $t$ goes to infinity, we easily obtain that

$$
\lim _{t \rightarrow \infty} \frac{1}{T} \int_{t}^{t+T} \beta^{+}(\tau) d \tau=0
$$

By Lemma 2.5, and since we are assuming that $2-c-c^{\prime}>0$, we get that

$$
\begin{aligned}
\limsup _{t \rightarrow \infty} \frac{1}{T} \int_{t}^{t+T} \alpha^{-}(\tau) d \tau & \leq \frac{c_{2} c_{5} h^{2}}{\nu c} \limsup _{t \rightarrow \infty} \frac{1}{T} \int_{t}^{t+T}|A u(\tau)|^{2} d \tau \\
& \leq \frac{2 c_{2} c_{5} h^{2} F^{2}}{\nu^{3} c}<\infty .
\end{aligned}
$$

Finally, by using Lemma 2.5 once again, we get that

$$
\liminf _{t \rightarrow \infty} \frac{1}{T} \int_{t}^{t+T} \alpha(\tau) d \tau \geq \frac{2-c^{\prime}-c}{c_{5} h^{2}} \nu-\frac{2 c_{2} c_{5} h^{2} F^{2}}{\nu^{3} c}>0,
$$

provided

$$
h^{-2} \geq \frac{\sqrt{2 c_{2}} c_{5} F}{\nu^{2} \sqrt{\left(2-c^{\prime}-c\right) c}} .
$$

Taking the value of $c$ that minimizes the right-hand side, namely, $c=1-c^{\prime} / 2$, and taking into account the definition of $h_{0}$ and the fact that $c_{5}=\left(1+c^{\prime} / 2\right) c_{1}$, we get

$$
h^{-2} \geq \frac{1+c^{\prime} / 2}{1-c^{\prime} / 2} h_{0}^{-2},
$$

which is always satisfied for some positive $c^{\prime}$ if $h<h_{0}$. Now, a simple application of Lemma 2.6 allows us to conclude that $\lim _{t \rightarrow \infty}\|w(t)\|=0$. This completes the proof of Theorem 2.1.

Remark. The identities (2.9), (2.10) have simplified the terms in Equation (2.15). Since, as pointed out earlier, these identities do not hold in the case of no-slip boundary conditions, one would have to estimate more terms in Equation (2.15) for the no-slip boundary condition case. As a consequence the estimate for the number of degrees of freedom will be larger. This is the essential difference between the periodic and no-slip Dirichlet boundary conditions.

\section{VARIOUS PARAMETERIZATIONS OF INERTIAL MANIFOLDS}

3.1. Background on inertial manifolds. In this section, we consider sets of determining degrees of freedom for evolution equations on a separable Hilbert space $H$ (with inner product $(\cdot, \cdot)$ and norm $|\cdot|$ ) of the form

$$
\frac{d u}{d t}+A u+R(u)=f
$$

that possess inertial manifolds. The space $H$ need not be infinite dimensional; however, for almost all of the physically interesting evolution equations, $H$ is infinite dimensional. The operator $A$ is assumed to be a positive, self-adjoint operator with 
compact inverse. The space $H$ will have a basis of eigenfunctions of the operator $A$. Let us denote $\left\{w_{j}\right\}_{j=1}^{\infty}$ the orthonormal basis for the space $H$ of eigenfunctions. That is, $A w_{j}=\lambda_{j} w_{j}$, where $0<\lambda_{1} \leq \lambda_{2} \leq \cdots \leq \lambda_{j} \leq \cdots$. By dissipative evolution equation we mean that the solutions of (3.1) eventually become bounded in $\mathcal{D}(A)$. That is, there exists a $\rho>0$ such that for every $u_{0} \in H$, there exists a time $T\left(u_{0}\right)$ such that

$$
|A u(t)| \leq \rho \quad \text { for all } t \geq T\left(u_{0}\right),
$$

where $u(t)$ is a solution of (3.1) with initial data $u_{0}$.

An inertial manifold for an evolution equation is a smooth finite-dimensional manifold (at least Lipschitz) that is positively invariant under the solution operator and exponentially attracts all solutions, [11]. Under certain general assumptions on the nonlinearity $R(u)$ and the linear operator $A$ one can guarantee the existence of the inertial manifold for the abstract equation (3.1); see [12]. Examples of such equations include the Kuramoto-Sivashinsky equation, the complex GinzburgLandau equation, the Cahn-Hilliard equation and certain reaction-diffusion equations; see, for example, [5], [9], [11], [29], [31] and the references therein. To date the existence of an inertial manifold for the two-dimensional NSE is unknown.

We assume in this section that (3.1) satisfies the general sufficient conditions mentioned in [12], and has an inertial manifold representable as the graph of a Lipschitz function $\Phi: P_{m} H \rightarrow\left(I-P_{m}\right) \mathcal{D}(A)$, where $P_{m}$ is the orthogonal projection of $H$ onto the span $\left\{w_{1}, \ldots w_{m}\right\}$.

More specifically, there exists a constant $l$ such that

$$
\left|A\left(\Phi\left(p_{1}\right)-\Phi\left(p_{1}\right)\right)\right| \leq l\left|A\left(p_{1}-p_{2}\right)\right| \quad \forall p_{1}, p_{2} \in P_{m} H .
$$

Moreover, Foias, Sell and Titi [12] have shown that the inertial manifold enjoys the so called exponential tracking property (the asymptotic completeness property). Though the asymptotic completeness property is shown to hold in a weaker topology than the one we need here, one may use a stronger version of the strong squeezing property given in [23] and the same proof of the asymptotic completeness property given in [12] to obtain the following

Proposition 3.1. For every solution $u(t)$, of $(3.1)$, there exists a time $T^{*}(|u(0)|)>$ 0 and a solution $u_{\mathcal{M}}(t)$ on the inertial manifold such that

$$
\left|A\left(u\left(t+T^{*}\right)-u_{\mathcal{M}}(t)\right)\right| \leq C \exp \left(-\frac{1}{2} \lambda_{m+1} t\right) \quad \forall t>0,
$$

for some positive constant $C$ which depends on $|u(0)|$ and $\rho$.

Once more, we associate to our set of degrees of freedom $\left\{\ell_{i}\right\}_{i=1}^{N}$, where $\ell_{i}$ are linear bounded functionals on $\mathcal{D}\left(A^{\gamma}\right)$, for some fixed $\gamma \in(0,1]$, the operator $R^{h}$ of the form

$$
R^{h}(u)=\sum_{i=1}^{N} \ell_{i}(u) \phi_{i},
$$

where now $\phi_{i}$ belongs to $H$. As in the preceding section, we restrict ourselves to those operators $R^{h}$ which are a good approximation of the inclusion map from $\mathcal{D}\left(A^{\gamma}\right)$ into $H$. More precisely, we require the following approximation inequality to hold:

$$
\left|u-R^{h} u\right| \leq c_{6} h^{\beta}\left|A^{\gamma} u\right|, \quad \forall u \in \mathcal{D}\left(A^{\gamma}\right),
$$


for some positive number $\beta$, and positive constant $c_{6}$.

We are now ready to state the main result of this section, which generalizes the result of [15] for the determining nodes and volume elements.

Theorem 3.2. Suppose that (3.1) has an inertial manifold $\mathcal{M}$ representable as the graph of a function $\Phi: P_{m} H \rightarrow\left(I-P_{m}\right) \mathcal{D}(A)$ and that $\Phi$ satisfies (3.3) and Proposition 3.1. Suppose that the approximation inequality (3.6) is satisfied and assume $h$ is small enough such that

$$
h<\left(c_{6}(1+l) \lambda_{m+1}^{\gamma}\right)^{-1 / \beta} .
$$

Then (i) The map $R^{h}: \mathcal{M} \rightarrow \operatorname{span}\left\{\phi_{1}, \cdots, \phi_{N}\right\}$ is a Lipschitz homeomorphism between $\mathcal{M}$ and $R^{h}(\mathcal{M})$. That is, there exist positive constants $c_{7}, c_{8}$ such that

$$
c_{7}\left|u_{1}-u_{2}\right| \leq\left|R^{h}\left(u_{1}\right)-R^{h}\left(u_{2}\right)\right| \leq c_{8}\left|u_{1}-u_{2}\right| \text { for all } u_{1}, u_{2} \in \mathcal{M} .
$$

(ii) Let $u(0), v(0) \in \mathcal{M}$, and assume that for some $t_{0} \geq 0$ we have

$$
R^{h} u\left(t_{0}\right)=R^{h} v\left(t_{0}\right) \text {. }
$$

Then

$$
u(t)=v(t) \quad \text { for all } t \geq 0 .
$$

(iii) The set of degrees of freedom $\left\{\ell_{i}\right\}_{i=1}^{N}$ is determining for (3.1).

Property (i) in the above theorem states that the inertial manifold may be parameterized in a smooth fashion (Lipschitz) in terms of the degrees of freedom, (3.5), provided we take sufficiently many degrees of freedom so that (3.7) is satisfied. Property (ii) states that solutions on the inertial manifold have the property that if their interpolates or projections $R^{h}$ agree at a single instant, then the solutions are identical. Whether this property holds for dissipative PDEs that are not known to have an inertial manifold, such as the Navier-Stokes equations, remains an open question. The number of determining degrees of freedom as stated in property (iii) is proportional to the dimension of the inertial manifold and is a consequence of the proof of the above theorem. However, with a different approach that depends on the equation, one might be able to obtain a much smaller estimate on the number of determining degrees of freedom. As an example we mention the one-dimensional complex Ginzburg-Landau equation, which is shown to have two determining nodes, [25], and the Kuramoto-Sivashinsky equation, which has four determining nodes, [7]. Both equations have inertial manifolds with a dimension that can be made arbitrarily large by adjusting the physical parameters appropriately.

The outline of the proof of the above theorem is in Section 3.3.

3.2. An example. To illustrate the content of the previous section, we consider the Kuramoto-Sivashinsky equation

$$
\begin{gathered}
\frac{\partial u}{\partial t}+\frac{\partial^{4} u}{\partial x^{4}}+\frac{\partial^{2} u}{\partial x^{2}}+u \frac{\partial u}{\partial x}=0, \\
u(0, x)=u_{0}(x), \\
u(t, x)=u(t, x+L) \quad L>0, t \geq 0,
\end{gathered}
$$

restricted to the invariant space of odd solutions. We take the Hilbert space $H=$ $\left\{u \in L^{2}((0, L)) \mid u(x)=u(x+L), u(x)=-u(L-x), x \in \mathbb{R}\right\}$. Here $A=\frac{\partial^{4}}{\partial x^{4}}$ with domain $\mathcal{D}(A)=H_{\text {per }}^{4}((0, L)) \cap H$, where $H_{\text {per }}^{m}(0, L)$ denotes the Sobolev space of 
functions that are periodic with period $L$ along with their first $m-1$ derivatives. The eigenvalues of $A$ are $\lambda_{j}=(2 \pi j / L)^{4}$ and eigenfunctions $w_{j}=\sin (2 \pi j / L)$.

We construct the operator $R^{h}$ on $\mathcal{D}\left(A^{1 / 2}\right)$, (i.e. $\left.\gamma=1 / 2\right)$ as follows. First, we divide the domain $(0, L]$ into $M$ equal disjoint elements each of width $h=L / M$, and set $x_{j}=j h$ for $1 \leq j \leq M$. Then we take $V^{h}=\operatorname{span}\left\{\phi_{i}, 1 \leq i \leq N=2 M\right\} \equiv$ $\operatorname{span}\left\{\phi_{1, j}, \phi_{2, j}, 1 \leq j \leq M\right\}$, where the functions $\phi_{1, i}$ and $\phi_{2, i}$ are piecewise-cubic $C^{1}([0, L])$ functions such that $\phi_{1, i}\left(x_{j}\right)=\delta_{i j}, \phi_{2, i}\left(x_{j}\right)=0$, and $\frac{\partial \phi_{1, i}}{\partial x}\left(x_{j}\right)=0$, $\frac{\partial \phi_{2, i}}{\partial x}\left(x_{j}\right)=\delta_{i j}$. These functions are the so-called Hermite cubics. Now, we define $R^{h}(u)$ to be the $L^{2}$-projection of $u$ into $V^{h}$. The operator $R^{h}$ thus constructed does satisfy the approximation inequality (3.6) with $\beta=2$, (see for example [34]).

For the existence of an inertial manifold for the Kuramoto-Sivashinsky equation the reader is referred to [11], or any of the references mentioned in the beginning of Section 3.1. Note that Theorem 3.2 requires that $M \sim \lambda_{m}^{\gamma / 2} \sim m$. This is the same order as the dimension of the inertial manifold which of course has dimension $m$.

3.3. Proof of Theorem 3.2. In this section we prove Theorem 3.2. To prove part (i) let $u_{1}, u_{2} \in \mathcal{M}$ and $w=u_{1}-u_{2}, p_{1}=P_{m} u_{1}, p_{2}=P_{m} u_{2}$. Using (3.3) and $0<\gamma \leq 1$, we have

$$
\begin{aligned}
\left|A^{\gamma} w\right| & =\left|A^{\gamma}\left(p_{1}-p_{2}+\Phi\left(p_{1}\right)-\Phi\left(p_{2}\right)\right)\right| \\
& \leq\left|A^{\gamma}\left(p_{1}-p_{2}\right)\right|+\lambda_{m+1}^{\gamma-1}\left|A\left(\Phi\left(p_{1}\right)-\Phi\left(p_{2}\right)\right)\right| \\
& \leq\left|A^{\gamma}\left(p_{1}-p_{2}\right)\right|+\lambda_{m+1}^{\gamma-1} l\left|A\left(p_{1}-p_{2}\right)\right| \\
& \leq(1+l) \lambda_{m+1}^{\gamma}|w| .
\end{aligned}
$$

Using (3.6) and the above, we obtain

$$
\begin{aligned}
|w| & \leq\left|R^{h} w\right|+c_{6} h^{\beta}\left|A^{\gamma} w\right| \\
& \leq\left|R^{h} w\right|+c_{6} h^{\beta}(1+l) \lambda_{m+1}^{\gamma}|w| .
\end{aligned}
$$

As a result we have

$$
|u-v| \leq L\left|R^{h}(u-v)\right|
$$

with $L=\left(1-c_{6} h^{\beta}(1+l) \lambda_{m+1}^{\gamma}\right)^{-1}$.

Similarly, from (3.6) and (3.9) we have that

$$
\begin{aligned}
\left|R^{h} w\right| & \leq|w|+c_{6} h^{\beta}\left|A^{\gamma} w\right| \\
& \leq\left\{1+c_{6} h^{\beta}(1+l) \lambda_{m+1}^{\gamma}\right\}|w| .
\end{aligned}
$$

This completes the proof of part (i).

To prove (ii) let $u(0)$ and $v(0)$ belong to the manifold $\mathcal{M}$. Since the inertial manifold is positively invariant, $u\left(t_{0}\right)$ and $v\left(t_{0}\right)$ also belong to $\mathcal{M}$ for all $t_{0} \geq 0$. By (3.10) we have that if $R^{h} u\left(t_{0}\right)=R^{h} v\left(t_{0}\right)$, then $u\left(t_{0}\right)=v\left(t_{0}\right)$. Since the reduction of the equation (3.1) to the inertial manifold gives an ordinary differential equation with Lipschitz nonlinearity, by the uniqueness of solutions we get that $u(t) \equiv v(t)$ for all $t \geq 0$.

To prove (iii), that is, the set of degrees of freedom $\left\{\ell_{i}\right\}_{i=1}^{N}$ is determining, let $u_{\mathcal{M}}$ and $v_{\mathcal{M}}$ be the two solutions on the inertial manifold corresponding to the solutions $u(t)$ and $v(t)$ respectively, that satisfy (3.4). As we mentioned the solutions are eventually bounded in $\mathcal{D}(A)$ and in particular in $\mathcal{D}\left(A^{\gamma}\right)$. Set $T^{*}=$ 
$\max \left\{T^{*}(|u(0)|), T^{*}(|v(0)|)\right\}$, where $\left.T^{*}(|u(0)|)\right), T^{*}(|v(0)|)$ are given in (3.4). Then for $t \geq T^{*}$ we have that

$$
\begin{aligned}
|u(t)-v(t)| \leq \quad \mid & R^{h}(u(t)-v(t))|+|\left(I-R^{h}\right)(u(t)-v(t)) \mid \\
\leq \quad \mid & R^{h}(u(t)-v(t)) \mid \\
& +\left|\left(I-R^{h}\right)\left(u(t)-u_{\mathcal{M}}\left(t-T^{*}\left(\left|u_{0}\right|\right)\right)\right)\right| \\
& +\left|\left(I-R^{h}\right)\left(v(t)-v_{\mathcal{M}}\left(t-T^{*}\left(\left|u_{0}\right|\right)\right)\right)\right| \\
& +\left|\left(I-R^{h}\right)\left(u_{\mathcal{M}}\left(t-T^{*}\left(\left|u_{0}\right|\right)\right)-v_{\mathcal{M}}\left(t-T^{*}\left(\left|v_{0}\right|\right)\right)\right)\right| .
\end{aligned}
$$

By (3.6), (3.9), and (3.4)

$$
\begin{aligned}
|u(t)-v(t)| \leq & \left|R^{h}(u(t)-v(t))\right|+2 C c_{6} h^{\beta} \lambda_{1}^{\gamma-1} \exp \left(-\frac{1}{2} \lambda_{m+1}\left(t-T^{*}\right)\right) \\
& +c_{6} h^{\beta}(1+l) \lambda_{m+1}^{\gamma}\left|u_{\mathcal{M}}\left(t-T^{*}\left(\left|u_{0}\right|\right)\right)-v_{\mathcal{M}}\left(t-T^{*}\left(\left|v_{0}\right|\right)\right)\right| .
\end{aligned}
$$

We estimate the quantity $\Theta=\left|u_{\mathcal{M}}\left(t-T^{*}\left(\left|u_{0}\right|\right)\right)-v_{\mathcal{M}}\left(t-T^{*}\left(\left|v_{0}\right|\right)\right)\right|$ by using

$$
\begin{aligned}
\Theta & \leq|u(t)-v(t)|+\left|u(t)-u_{\mathcal{M}}\left(t-T^{*}\left(\left|u_{0}\right|\right)\right)\right|+\left|v(t)-v_{\mathcal{M}}\left(t-T^{*}\left(\left|v_{0}\right|\right)\right)\right| \\
& \leq|u(t)-v(t)|+2 C \lambda_{1}^{-1} \exp \left(-\frac{1}{2} \lambda_{m+1}\left(t-T^{*}\right)\right)
\end{aligned}
$$

Since, by hypothesis, (3.7), $L=\left(1-c_{6} h^{\beta}(1+l) \lambda_{m+1}^{\gamma}\right)^{-1}$ is a positive number, we can easily combine the inequalities above to obtain

$$
\begin{aligned}
|u(t)-v(t)| \leq & L\left|R^{h}(u(t)-v(t))\right| \\
& +2 C c_{6} L \lambda_{1}^{-1} h^{\beta}\left[\lambda_{1}^{\gamma}+(1+l) \lambda_{m+1}^{\gamma}\right] \exp \left(-\frac{1}{2} \lambda_{m+1}\left(t-T^{*}\right)\right) .
\end{aligned}
$$

Thus, if $\ell_{i}(u(t)-v(t)), 1 \leq i \leq N$, go to zero as $t$ goes to infinity, then $\mid R^{h}(u(t)-$ $v(t)) \mid$ also goes to zero as $t$ goes to infinity. This shows that the set of degrees of freedom $\left\{\ell_{i}\right\}_{i=1}^{N}$ is determining. This completes the proof of Theorem 3.2.

\section{Degrees of freedom for general dissipative EVolution EQUations}

In this section we give sufficient conditions so that the evolution equation on a separable Hilbert space $H$

$$
\frac{d u}{d t}+A u+R(u)=f
$$

has a finite set of determining degrees of freedom $\left\{\ell_{i}\right\}_{i=1}^{N}$. The operator $A$ is assumed to be a positive, self-adjoint operator with compact inverse. Hence, as before there exists a basis $\left\{w_{j}\right\}_{j=1}^{\infty}$ of eigenfunctions of $A$. That is $A w_{j}=\lambda_{j} w_{j}$. We suppose that the degrees of freedom $\left\{\ell_{i}\right\}_{i=1}^{N}$ satisfy (3.5) and (3.6) of the previous section.

We emphasize that we do not assume in this section that (4.1) has an inertial manifold. When an inertial manifold exists for an evolution equation one can deduce, as was done in the previous section, that there exists a finite set of determining degrees of freedom. However, due to the nature of the proof that was given in Section 3.3, the number of degrees of freedom is of the order of the dimension of the inertial manifold.

Given a specific equation alternate proofs may show that the number of degrees of freedom is much smaller than the bound derived in the previous section. Thus 
the number of determining degrees of freedom need not be related to the dimension of the inertial manifold at all. See the discussion below Theorem 3.2.

A dissipative evolution equation of the form (4.1) may still have a finite set of determining degrees of freedom - regardless of the existence of an inertial manifold. We demonstrate this below. In general the estimate we derive on $h$ for the degrees of freedom to be determining may be improved for a given equation by taking advantage of the properties of the nonlinear term $R(u)$ specific to that equation (as we did for the NSE in Section 2) as well as the regularity of the solutions as for the one-dimensional complex Ginzburg-Landau equation, [25], and the KuramotoSivashinsky equation, [7]. Here we will not try to make our estimates as sharp as possible.

We make two assumptions about (4.1). The first is that the system is dissipative in $\mathcal{D}(A)$ in the sense of (3.2). We denote by $B(\rho)$ the set $\{u \in \mathcal{D}(A):|A u| \leq \rho\}$. Second, we suppose the nonlinear term satisfies

$$
|(R(u)-R(v), A(u-v))| \leq K(\rho)|(u-v)|^{r_{1}}\left|A^{\theta}(u-v)\right|^{r_{2}}, \quad \text { for all } u, v \in B(\rho),
$$

where $\theta \in[0,1]$ and where $r_{1}>0, r_{2} \geq 0$ are real numbers and are such that $r_{1}+r_{2}=2$. These two assumptions hold for a variety of dissipative evolution equations, including the ones mentioned at the beginning of Section 3 as well as for the NSE.

Theorem 4.1. Suppose that $\left\{\ell_{i}\right\}_{i=1}^{N}$ satisfies (3.5) and (3.6) in Section 3. Then under the above assumptions on (4.1), $\left\{\ell_{i}\right\}_{i=1}^{N}$ is a set of determining degrees of freedom provided that $h$ is sufficiently small so that

$$
1-K(\rho) k_{1} \lambda_{1}^{r_{1} \gamma+r_{2} \theta-2}\left(c_{6} h^{\beta}\right)^{r_{1}}>0
$$

holds. The constant $k_{1}$ satisfies $(a+b)^{r_{1}} \leq k_{1}\left(a^{r_{1}}+b^{r_{1}}\right)$ for all $a, b \in \mathbb{R}^{+}$. That is, $k_{1}=1$ if $r_{1} \leq 1$, and $k_{1}=2^{r_{1}-1}$ if $r_{1} \geq 1$.

Proof. Suppose, without loss of generality, that $u(t)$ and $v(t)$ solve (4.1) and satisfy $u(t), v(t) \in B(\rho)$ for all $t \geq 0$. Set $w(t)=u(t)-v(t)$. By assumption $\left|R^{h} w(t)\right| \rightarrow 0$ as $t \rightarrow \infty$. Subtracting the equations governing $u(t), v(t)$, taking the inner product with $A w$ and using (4.2), we find that

$$
\frac{1}{2} \frac{d}{d t}\|w\|^{2}+|A w|^{2} \leq K(\rho)|w|^{r_{1}}\left|A^{\theta} w\right|^{r_{2}},
$$

where $\|\cdot\|=\left|A^{1 / 2} \cdot\right|$. From (3.6) and our assumptions on $r_{1}, r_{2}$, and the inequality $\left|A^{\gamma} u\right| \leq \lambda_{1}^{\gamma-1}|A u|$ for all $u \in \mathcal{D}(A)$ ( $\gamma$ is given in (3.6)), we have that

$$
\begin{aligned}
\frac{1}{2} \frac{d}{d t}\|w\|^{2}+|A w|^{2} \leq & K(\rho)\left(\left|R^{h} w\right|+c_{6} h^{\beta}\left|A^{\gamma} w\right|\right)^{r_{1}}\left|A^{\theta} w\right|^{r_{2}} \\
\leq & K(\rho) \rho^{r_{2}} \lambda_{1}^{(\theta-1) r_{2}} k_{1}\left|R^{h} w\right|^{r_{1}} \\
& +K(\rho) k_{1} \lambda_{1}^{r_{1} \gamma+r_{2} \theta-2}\left(c_{6} h^{\beta}\right)^{r_{1}}|A w|^{2} .
\end{aligned}
$$

Using our assumption on $h^{\beta},(4.3)$, the inequality $\lambda_{1}^{1 / 2}\|w\| \leq|A w|$ and Gronwall's inequality, the result follows. 


\section{ACKNOWLEDGMENTS}

E.S.T. gratefully acknowledges the hospitality of the Institute for Geophysics and Planetary Physics (IGPP) and the Center for Nonlinear Studies (CNLS) at Los Alamos National Laboratory. D.A.J. was partially supported by the Department of Energy (CHAMMP) research program. This work was also supported in part by the Joint University of California - Los Alamos National Laboratory (INCOR) program. E.S.T. was supported in part by the NSF Grant No. DMS-93-08774, ONR contract No. N00014-94-C-0024 at BEAM Engineering and Applied Research, Ithaca, NY, and by the University of California-Irvine Faculty Research Fellowship.

\section{REFERENCES}

1. A. V. BABIN AND M. I. VishiK, Attractors of partial differential equations and estimates of their dimension, Uspekhi Mat. Nauk, 38, (1983), 133-187 (in Russian); Russian Math. Surveys, 38, 151-213 (in English). MR 84k:58133

2. P. Ciarlet, The Finite Element Method for Elliptic Problems, North-Holland, 1978. MR 58:25001

3. B. Cockburn, D.A. Jones, E.S. Titi, Degrés de liberté déterminants pour équations nonlinéaires dissipatives, C.R. Acad. Sci.-Paris, Sér. I, 321, (1995), 563-568.

4. P. Constantin, C. Foias, Navier-Stokes Equations, University of Chicago Press, 1988. MR 90b: 35190

5. P. Constantin, C. Foias, B. Nicolaenko, R. Temam, Integral Manifolds and Inertial Manifolds for Dissipative Partial differential Equations, Applied Mathematics Sciences, 70, Springer-Verlag, 1989. MR 90a:35026

5.5. P. Constantin, C. Foias, R. Temam, On the large time Galerkin approximation of the Navier-Stokes equations, SIAM J. Numer. Anal. 21 (1984), 615-634. MR 85i:65145

6. P. Constantin, C. Foias, R. Temam, On the dimension of the attractors in two-dimensional turbulence, Physica, D30, (1988), 284-296. MR 89j:76056

7. C. Foias, I. KukAVICA, Determining nodes for the Kuramoto-Sivashinsky equation, J. Dynam. Diff. Eq., 7 (1995), 365-373. MR 96b:35190

8. C. Foias, O.P. Manley, R. Temam, Y. Treve, Asymptotic analysis of the Navier-Stokes equations, Physica, D9, (1983), 157-188. MR 85e:35097

9. C. Foias, B. Nicolaenko, G.R. Sell, R. Temam, Inertial manifolds for the KuramotoSivashinsky equation and an estimate of their lowest dimensions, J. Math. Pures Appl., 67, (1988), 197-226. MR 90e:35137

10. C. FoiAs, G. Prodi, Sur le comportement global des solutions non stationnaires des équations de Navier-Stokes en dimension two, Rend. Sem. Mat. Univ. Padova, 39, (1967), 1-34. MR 36:6764

11. C. Foias, G. Sell, R. Temam, Inertial manifolds for nonlinear evolutionary equations, J. Diff. Eq., 73, (1988), 309-353. MR 89e:58020

12. C. FoiAs, G. Sell, E.S. Titi, Exponential tracking and approximation of inertial manifolds for dissipative nonlinear equations, J. Dynam. and Diff. Eq., 1, No. 2, (1989), 199-243. MR 90k:35031

13. C. Foias, R. Temam, Asymptotic numerical analysis for the Navier-Stokes equations, Nonlinear Dynamics and Turbulence, Edit. by Barenblatt, Iooss, Joseph, Boston: Pitman Advanced Pub. Prog., 1983. CMP 16:17

14. C. Foias, R. Temam, Determination of the solutions of the Navier-Stokes equations by a set of nodal values, Math. Comput., 43, (1984), 117-133. MR 85f:35165

15. C. Folas, E.S. Titi, Determining nodes, finite difference schemes and inertial manifolds, Nonlinearity, 4, (1991), 135-153. MR 92a:65241

16. V. Girault, P.A. Raviart, Finite Element Approximations of the Navier-Stokes Equations, Lecture Notes in Mathematics, 749, Springer Verlag, 1979. MR 83b:65122

17. J.K. Hale, X.-B. Lin, G. Raugel, Upper Semicontinuity of Attractors for Approximations of Semigroups and Partial Differential Equations. Math. Comp., 50, (1988), 89-123. MR 89a: 47093 
18. J.G. Heywood, R. RANNACHER, Finite element approximation of the nonstationary NavierStokes problems. Part II Stability of solutions and error estimates uniform in time, SIAM J. Num. Anal., 23, (1986), 750-777. MR 88b:65132

19. D.A. Jones, A.M. Stuart, E.S. Titi, Persistence of invariant sets for dissipative evolution equations, (submitted).

20. D.A. Jones, E.S. Titi, On the number of determining nodes for the $2 D$ Navier-Stokes equations, J. Math. Anal. Appl., 168, (1992), 72-88. MR 93f:35179

21. D.A. Jones, E.S. Titi, Determining finite volume elements for the $2 D$ Navier-Stokes equations, Physica, D60, (1992), 165-174. MR 93j:35133

22. D.A. Jones, E.S. Titi, Upper bounds on the number of determining modes, nodes, and volume elements for the Navier-Stokes equations, Indiana Math. J., 42, (1993), 875-887. MR 94k:35249

23. D.A. Jones, E.S. Titi, $C^{1}$ Approximations of inertial manifolds for dissipative nonlinear equations, J. Diff. Eq., 127, (1996), 54-86. CMP 96:12

24. R.H. Kraichnan, Interial ranges in two-dimensional turbulence, Phys. Fluids, 10, (1967), $1417-1423$.

25. I. Kukavica, On the number of determining nodes for the Ginzburg-Landau equation, Nonlinearity, 5, (1992), 997-1006. MR 93h:35108

26. L. Landau, E. Lifschitz, Fluid Mechanics, Addison-Wesley, New-York, 1953.

27. J.L. Lions, Quelques Méthodes de Résolution de Problèmes aux Limites Non Linéaires, Dunod, Paris, 1969. MR 41:4326

28. V.X. LiU, A sharp lower bound for the Hausdorff dimension of the global attractors of the $2 D$ Navier-Stokes equations, Commun. Math. Phys., 158, (1993), 327-339. MR 94k:35251

29. J. Mallet-Paret, G. Sell, Inertial manifolds for reaction diffusion equations in higher space dimensions, J. Amer. Math. Soc., 1, (1988), 805-866. MR 90h:58056

30. R. Temam, Navier-Stokes Equations and Nonlinear Functional Analysis, CBMS Regional Conference Series, No. 41, SIAM, Philadelphia, 1983. MR 86f:35152

31. R. Temam, Infinite Dimensional Dynamical Systems in Mechanics and Physics, Springer Verlag, New York, 1988. MR 89m:58056

32. E.S. Titi, On a criterion for locating stable stationary solutions to the Navier-Stokes equations, Nonlinear Anal. TMA, 11, (1987), 1085-1102. MR 89f:35173

33. E.S. Titi, Un critère pour l'approximation des solutions périodiques des équations de NavierStokes, C.R. Acad. Sci. Paris, 312, Série I. No. 1, (1991), 41-43. MR 92e:35130

34. R. Wait, A.R. Mitchell, Finite Element Analysis and Applications, John Wiley \& Sons, 1985. MR 87i: 65192

School of Mathematics, University of Minnesota, Minneapolis, Minnesota 55455

E-mail address: cockburn@math.umn.edu

IGPP, University of California, los Alamos National laboratory, Mail Stop C305, Los Alamos, New Mexico 87544

E-mail address: dajones@kokopelli.lanl.gov

Department of Mathematics, and Department of Mechanical and Aerospace EngiNeEring, University of California, Irvine, California 92697

E-mail address: etiti@math.uci.edu 